NASA Contractor Report 204142

$N \cdot 34$
$0610 \% 3$

ICOMP-97-09; CMOTT-97-04

AIAA-96-0382

\title{
An NPARC Turbulence Module With Wall Functions
}

\section{J. Zhu and T.-H. Shih}

Institute for Computational Mechanics in Propulsion and Center for Modeling of Turbulence and Transition

Cleveland, Ohio

August 1997

Prepared for

Lewis Research Center

Under Cooperative Agreement NCC3-370

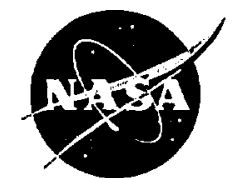

National Aeronautics and Space Administration

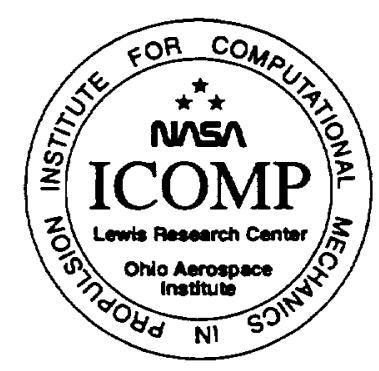


. 


\title{
AN NPARC TURBULENCE MODULE WITH WALL FUNCTIONS
}

\author{
J. Zhu * and T.-H. Shih \\ Center for Modeling of Turbulence and Transition \\ ICOMP, OAI, NASA Lewis Research Center \\ Cleveland, Ohio 44135
}

\begin{abstract}
The turbulence module recently developed for the NPARC code has been extended to include wall functions. The Van Driest transformation is used so that the wall functions can be applied to both incompressible and compressible flows. The module is equipped with three two-equation $K-\epsilon$ turbulence models: Chien, Shih-Lumley and CMOTT models. Details of the wall functions as well as their numerical implementation are reported. It is shown that the inappropriate artificial viscosity in the near-wall region has a big influence on the solution of the wall function approach. A simple way to eliminate this influence is proposed, which gives satisfactory results during the code validation. The module can be easily linked to the NPARC code for practical applications.
\end{abstract}

\section{Introduction}

A turbulence module (Zhu and Shih, 1995) has been developed for the NPARC code. The module is written in a self-contained manner so that the user can use any turbulence model built in the module without concern as to how it is implemented and solved. The input to the module is the mean flow variables, boundary and geometric information which are to be provided by the NPARC code. The output of the module is the turbulent eddy-viscosity $\mu_{t}$ and/or relevant turbulent source terms which are needed for the mean flow calculation. The interaction between the NPARC code and the turbulence module will give the final turbulent flow solution. The turbulence module is conceived as a vehicle to facilitate the technology transfer from the model development to the model application. With the module, any development in the turbulence modeling can be quickly and directly validated by and applied to

\footnotetext{
- Member AIAa
}

calculations of flows of engineering interests.

So far, three low Reynolds number two-equation turbulence models have been built into the module: Chien (1982), Shih and Lumley (1993) and CMOTT realizable models (Yang et al., 1995; Zhu and Shib, 1995). Unlike the Chien's model, both the ShihLumley and CMOTT models do not involve the dimensionless wall distance $y^{+}$, an advantage for separated flow calculations. The module with these turbulence models has been applied to a number of flows including flows over a flat plate, in an ejector nozzle, in a transonic diffuser, and a boat-tail nozzle flow (Yang et al., 1995). For all the flow cases tested so far, it has been found that both the ShihLumley and CMOTT models produce improved or similar predictions compared with the Chien model. The CMOTT model with variable $C_{\mu}$ turns out to be more computationally robust than the other two. It was able to give numerical solutions in cases where the models with constant $C_{\mu}$ suffered from numerical instability.

The major problems or difficulties associated with the low Reynolds number turbulence models are: 1) They require very fine grid spacing in nearwall regions, thus increasing considerably computational burden, especially in three-dimensional cases. Moreover, the highly stretched nature of mesh distribution may have an adverse impact on numerical stability. 2) Most of models are not of tensorial invariant form, that is, they contain a distance parameter normal to the wall. The wall-distance dependency causes inconvenience for model applications in complicated geometries. Currently, great effort is being given in the area of near-wall turbulence modeling to remove this dependency, but no satisfactory result has been obtained yet. 3) Most of the low Reynolds number turbulence models were finetuned against attached flows, which is, of course, not sufficient to guarantee their good performance for separated flows.

An alternative is to use the high Reynolds number turbulence models. Here, the governing equations are integrated to a point far outside the vis- 
cous sublayer rather than down to the wall, and the near-wall region is bridged over with the wall functions. Although in theory the wall function approach is only valid for certain attached flows with no pressure gradient and mass transfer, it has been applied in practice to many separated flows with varing degree of success. For those flows where maximum shear stresses occur far away from the wall, the near-wall turbulence modeling is not critical for overall flow simulations. In these cases, use of the wall functions has a very beneficial effect on the stability and economy of computations. Although the principle argument for originally adopting the wall function approach (economy of grid points) has been weakened as larger and faster computers have become available, it will still find its applications in predicting complex flows, especially for large scale engineering problems.

In the present work, we extend the turbulence module by including the wall functions. For incompressible flows, the universal law of the wall may be expressed as

$$
\frac{U}{U_{\tau}}=\frac{1}{\kappa} \ln \left(\frac{\rho U_{\tau} y}{\mu}\right)+C
$$

where $\kappa=0.41$ and $C=5.2$. The derivation of Equation (1) is based on the assumption that the shear stress in the region close to the wall is constant and equal to the wall shear stress. It has been shown (Viegas et al., 1985; Huang and Coakdey, 1993) that the same form also exists for compressible flows with the velocity $U$ being replaced by the Van Driest transformed velocity $U_{c}$ (Van Driest, 1951). For the $K-\epsilon$ turbulence models, the convection and diffusion terms of their transport equations are negligible in the inertial sublayer so that local equilibrium prevails, which implies that the production of the turbulent hinetic energy $K$ is equal to the dissipation rate $\epsilon$ of $K$. The local equilibrium condition leads to two simple relations which can be used as boundary conditions for $K$ and $\epsilon$ for both incompressible and compressible flows. The compressible wall functions have been successfully applied to both attached and separated flows under Mach number ranging from 0.1 to 10 (Huang and Coakley, 1993).

Although the wall functions look simple, their numerical implementation is not trivial. The main difficulty comes from the logarithmic law in which both $U$ and $U_{T}$ are unknown, and $U_{\tau}$ cannot explicitly be solved for. It is prone to being numerically unstable if one uses Equation (1) and iteratively solves $U_{r}$ to obtain the boundary conditions for the Navier-Stokes equations. In this work, we use an implicit procedure which directly incorpo- rates Equation (1) into the Navier Stokes equations. In this way, there is no need to solve Equation (1) for $U_{\tau}$ by sub-iteration. The implicit method turns out to be more stable than the explicit one. Another important issue is the artificial viscosity. Chitsomboon (1994) found that the artificial viscosity originally implemented in the NPARC code totally spoiled the solution of the wall functions. This was because the artificial viscosity became unrealistically large in the vicinity of walls due to very steep velocity gradients resulting from the coarseness of grid spacing as required by the wall function approach. He fixed up this problem by extrapolating velocities at the wall rather than using the physical values of no-slip velocities, when calculating the artificial viscosity. In the present work, we simply turn off the artificial viscosity in the near-wall region.

In what follows, we will present the details of the wall functions and their implementation, and demonstrate how to link the turbulence module to the NPARC code. Detailed applications will be reported in another paper.

\section{Calculation Approach}

The following presentation is only restricted to the numerical aspects related to the implementation of the wall functions. Refer to Cooper and Sirbaugh (1989 and 1990) about the details of the NPARC code and to Zhu and Shih (1995) about the details of the turbulence module.

\subsection{Governing Equations}

In the NPARC code, the following nondimensional, Reynolds-averaged Navier-Stokes equations are solved:

$$
\frac{\partial \hat{Q}}{\partial t}+\frac{\partial \hat{F}_{1}}{\partial \xi}+\frac{\partial \hat{F}_{2}}{\partial \eta}=\frac{\partial \hat{G}_{1}}{\partial \xi}+\frac{\partial \hat{G}_{2}}{\partial \eta}
$$

where $\hat{Q}, \hat{F}_{j}$ and $\hat{G}_{j}(j=1,2)$ are the conservation variable vector, inviscid flux vectors and viscous flux vectors, respectively. Because only the viscous flux vectors need to be modified with the use of the wall functions, their detailed forms are given below:

$$
\hat{G}_{1}=\left[\begin{array}{l}
g_{11} \\
g_{12} \\
g_{13} \\
g_{14}
\end{array}\right], \quad \hat{G}_{2}=\left[\begin{array}{l}
g_{21} \\
g_{22} \\
g_{23} \\
g_{24}
\end{array}\right]
$$




$$
\begin{aligned}
& g_{11}=0 \\
& g_{12}=\hat{\tau}_{11}=J^{-1}\left(\xi_{x} \tau_{11}+\xi_{y} \tau_{12}\right) \\
& g_{13}=\hat{\tau}_{12}=J^{-1}\left(\xi_{x} \tau_{21}+\xi_{y} \tau_{22}\right) \\
& g_{14}=U \hat{\tau}_{11}+V \hat{\tau}_{12}-J^{-1}\left(\xi_{x} q_{1}+\xi_{y} q_{2}\right) \\
& g_{21}=0 \\
& g_{22}=\hat{\tau}_{21}=J^{-1}\left(\eta_{x} \tau_{11}+\eta_{y} \tau_{12}\right) \\
& g_{23}=\hat{\tau}_{22}=J^{-1}\left(\eta_{x} \tau_{21}+\eta_{y} \tau_{22}\right) \\
& g_{24}=U \hat{\tau}_{21}+V \hat{\tau}_{22}-J^{-1}\left(\eta_{x} q_{1}+\eta_{y} q_{2}\right) \\
& \tau_{11}=\frac{\mu_{t o t}}{R_{e}}\left[2 \frac{\partial U}{\partial x}-\frac{2}{3}\left(\frac{\partial U}{\partial x}+\frac{\partial V}{\partial y}\right)\right] \\
& \tau_{22}=\frac{\mu_{t o t}}{R_{e}}\left[2 \frac{\partial V}{\partial y}-\frac{2}{3}\left(\frac{\partial U}{\partial x}+\frac{\partial V}{\partial y}\right)\right] \\
& \tau_{12}=\tau_{21}=\frac{\mu_{t o t}}{R_{e}}\left(\frac{\partial U}{\partial y}+\frac{\partial V}{\partial x}\right) \\
& q_{1}=-\alpha \frac{\partial T}{\partial x}, \quad q_{2}=-\alpha \frac{\partial T}{\partial y} \\
& \alpha=\frac{1}{(\gamma-1) R_{e}}\left(\frac{\mu}{P_{r}}+\frac{\mu_{t}}{P_{r t}}\right)
\end{aligned}
$$

In the turbulence module developed for the NPARC code, the three $K-\epsilon$ turbulence models have been implemented: Chien, Shih-Lumley and CMOTT models. The detailed forms of these models are given in Zhu and Shih (1995).

\subsection{Wall Functions}

The compressible law of the wall (Huang and Coakley, 1993) is used in the turbulence module. Following the NPARC nondimensionalization, this law can be written as

$$
U_{c}^{+}=\frac{U_{c}}{U_{\tau}}=\frac{1}{\kappa} \ln \left(E y^{+}\right)
$$

where

$$
\begin{aligned}
& U_{\tau}=\sqrt{(\tau / \rho)_{\text {wall }}} \\
& y^{+}=R_{e} U_{\tau} y(\rho / \mu)_{w a l l} \\
& \kappa=0.41, \quad E=8.4317
\end{aligned}
$$

and $U_{c}$ is the Van Driest transformed velocity defined by (Van Driest, 1951):

$$
U_{c}=\sqrt{B}\left[\sin ^{-1}\left(\frac{A+U}{D}\right)-\sin ^{-1}\left(\frac{A}{D}\right)\right]
$$

where

$$
\begin{aligned}
& A=\left(\frac{q}{\tau}\right)_{\text {wall }} \\
& B=\frac{2 T_{\text {wall }}}{(\gamma-1) P_{\tau t}} \\
& D=\sqrt{A^{2}+B}
\end{aligned}
$$

In the near-wall region, with the convection neglected the energy equation can be reduced to give an expression for the total heat flux

$$
q=q_{t w a l l}+U \tau
$$

and with the local equilibrium assumption (Launder and Spalding, 1974), the turbulent kinetic energy $K$ and its dissipation rate $\epsilon$ can be calculated by

$$
\begin{gathered}
K=\frac{\tau_{w a l l} / \rho}{0.3} \\
\epsilon=\frac{\left(\tau_{w a l l} / \rho\right)^{3 / 2}}{\kappa y}
\end{gathered}
$$

In the above expressions, the subscript wall refers to the value of the corresponding function at the wall. Equations (10) and (14) - (16) form the wall functions which are used to bridge over the first grid point and the solid wall.

\subsection{Numerical Implementation}

From Equations (10) and (11), the near-wall shear stress can be written as

$$
\tau=\tau_{\text {wall }}=\rho_{w \text { all }} U_{\tau} \frac{U_{c}}{U_{c}^{+}}=\frac{\mu_{t}}{R_{e}} \frac{U}{y}
$$

where $\mu_{t}$ is an effective turbulent viscosity connecting the wall and the first grid point

$$
\mu_{t}=\frac{y^{+} \mu_{w a l l} U_{c}}{U U_{c}^{+}}
$$

An advantage of Equation (17) in calculating separated flows is worthy of note: the direction of the wall shear stress $\tau_{\text {wall }}$ is determined by that of the flow velocity $U$ while $\tau_{w a l l}$ calculated from Equation (10) or (1) can only have a positive sign.

From Equation (17), the general form of shear stress at the first grid point can be expressed as

$$
\vec{\tau}=\vec{\tau}_{w a l l}=\lambda \vec{U}_{t}
$$

where 


$$
\lambda= \begin{cases}\mu_{t} /\left(R_{e} \Delta n\right) & \text { if } y^{+}>11.6 \\ \mu_{w a l l} /\left(R_{e} \Delta n\right) & \text { otherwise }\end{cases}
$$

$\vec{U}_{t}$ and $\Delta n$ are the tangential component of the resultant velocity and the normal distance from the wall, respectively. Equations (19) and (20) simply treat the near-wall region as a laminar sublayer $\left(y^{+}<11.6\right)$ and a fully turbulent layer $\left(y^{+}>11.6\right)$. This treatment prevents the wall function procedure from producing abnormal results when $y^{+}$tends to zero, such as in the vicinity of separation or reattachment points.

Similarly, the total heat flux at the first grid point can be written as

$$
q=-\alpha\left(T-T_{\text {wall }}\right)
$$

where

$$
\alpha= \begin{cases}\lambda /\left[(\gamma-1) P_{r t}\right] & \text { if } y^{+}>11.6 \\ \lambda /\left[(\gamma-1) P_{r}\right] & \text { otherwise }\end{cases}
$$

and the heat flux at the wall can be calculated by

$$
q_{w a l l}=-\alpha\left(T-T_{w a l l}\right)-\vec{U}_{t} \cdot \vec{\tau}_{w a l l}
$$

Consider first the wall of $\eta=$ constant. In this case, only the viscons flux vector $\hat{G}_{2}$ in Equation (2) needs to be modified with the use of the wall functions. In the NPARC code, it is calculated by

$$
\begin{aligned}
& \frac{\partial g_{22}}{\partial \eta}=g_{22, n}-g_{22,,} \\
& \frac{\partial g_{2 s}}{\partial \eta}=g_{23, n}-g_{23, s} \\
& \frac{\partial g_{24}}{\partial \eta}=g_{24, n}-g_{24,,}
\end{aligned}
$$

where the subscripts $s$ and $n$ refer to the sonth and north faces of the control volume under consideration (Figure 1). From Equations (5) - (7), the components of the vector $\hat{G}_{2}$ can be written as

$$
\begin{aligned}
g_{32}= & \frac{\eta_{x}^{2}+\eta_{y}^{2}}{J} \frac{\mu_{t o t}}{R_{e}} \frac{\partial U}{\partial \eta}+\cdots \\
g_{23}= & \frac{\eta_{x}^{2}+\eta_{y}^{2}}{J} \frac{\mu_{t o t}}{R_{e}} \frac{\partial V}{\partial \eta}+\cdots \\
g_{24}= & U \hat{\tau}_{21}+V \hat{\tau}_{22} \\
& +\frac{\eta_{x}^{2}+\eta_{y}^{2}}{J} \alpha \frac{\partial T}{\partial \eta}+\cdots
\end{aligned}
$$

From geometrical consideration, we have

$$
\frac{\eta_{x}^{2}+\eta_{y}^{2}}{J}=\frac{J^{-2}\left(\eta_{x}^{2}+\eta_{y}^{2}\right)}{J-1}=\frac{\Delta l^{2}}{\Delta \Omega}=\frac{\Delta l}{\Delta n}
$$

where $\Delta \Omega$ and $\Delta l$ are the volume and face area of the control volume, respectively.

In the wall function approach, all the stresses acting on the cell face considered are replaced by the wall shear stress given by Equation (19). Therefore, for the south wall, Equations (25) are replaced by

$$
\begin{aligned}
g_{22, s}= & \Delta l \lambda U_{t x} \\
g_{23,4}= & \Delta l \lambda U_{t y} \\
g_{24, s}= & U g_{22,,}+V g_{23,\lrcorner} \\
& +\Delta l \alpha\left(T-T_{w}\right)
\end{aligned}
$$

where $U_{t x}$ and $U_{t y}$ are the $x$ - and $y$-components of the tangential velocity $\vec{U}_{t}$, respectively. If $n_{x}$ and $n_{y}$ are the Cartesian components of the unit normal vector at the wall, $U_{t z}$ and $U_{t y}$ can be calculated by

$$
\begin{aligned}
& U_{t x}=n_{y}^{2} U-n_{x} n_{y} V \\
& U_{t y}=n_{x}^{2} V-n_{x} n_{y} U
\end{aligned}
$$

Similarly, for the north wall, we have

$$
\begin{aligned}
g_{22, n}= & -\Delta l \lambda U_{t x} \\
g_{23, n}= & -\Delta l \lambda U_{t y} \\
g_{24, n}= & U g_{22, s}+V g_{23, s} \\
& -\Delta l \alpha\left(T-T_{w}\right)
\end{aligned}
$$

For the wall of $\xi=$ constant, only the viscous flux vector $\hat{G}_{1}$ in Equation (2) needs to be modified when using the wall functions. In the NPARC code, we have

$$
\begin{aligned}
& \frac{\partial g_{12}}{\partial \xi}=g_{12, e}-g_{12, w} \\
& \frac{\partial g_{13}}{\partial \xi}=g_{13, e}-g_{13, w} \\
& \frac{\partial g_{14}}{\partial \xi}=g_{14, e}-g_{14, w}
\end{aligned}
$$

where the subscripts $w$ and $e$ refer to the west and east faces of the control volume under consideration (Figure 1). From Equations (4), (6) and (7), the components of the vector $\hat{G}_{1}$ can be written as 


$$
\begin{aligned}
g_{12}= & \frac{\xi_{x}^{2}+\xi_{y}^{2}}{J} \frac{\mu_{t o t}}{R_{e}} \frac{\partial U}{\partial \xi}+\cdots \\
g_{13}= & \frac{\xi_{x}^{2}+\xi_{y}^{2}}{J} \frac{\mu_{t o t}}{R_{e}} \frac{\partial V}{\partial \xi}+\cdots \\
g_{14}= & U \hat{\tau}_{11}+V \hat{\tau}_{12} \\
& +\frac{\xi_{x}^{2}+\xi_{y}^{2}}{J} \alpha \frac{\partial T}{\partial \xi}+\cdots
\end{aligned}
$$

and the geometric consideration leads to

$$
\frac{\xi_{x}^{2}+\xi_{y}^{2}}{J}=\frac{J^{-2}\left(\xi_{x}^{2}+\xi_{y}^{2}\right)}{J-1}=\frac{\Delta l^{2}}{\Delta \Omega}=\frac{\Delta l}{\Delta n}
$$

where $\Delta \Omega$ and $\Delta l$ are the volume and face area of the control volume, respectively.

After replacing all the stresses acting on the cell face considered by the wall shear stress given by Equation (19), Equations (31) become:

for the west wall

$$
\begin{aligned}
g_{12, w}= & \Delta l \lambda U_{t x} \\
g_{13, w}= & \Delta l \lambda U_{t y} \\
g_{14, w}= & U g_{12, w}+V g_{13, w} \\
& +\Delta l \alpha\left(T-T_{w a l l}\right)
\end{aligned}
$$

for the east wall

$$
\begin{aligned}
g_{12, e}= & -\Delta l \lambda U_{t x} \\
g_{13, e}= & -\Delta l \lambda U_{t y} \\
g_{14, e}= & U g_{12, e}+V g_{13, e} \\
& -\Delta l \alpha\left(T-T_{w a l l}\right)
\end{aligned}
$$

The sequence in which the above equations are solved together with the Navier-Stokes and turbulence equations in the code is as follows:

a. Initialize all field values.

b. Calculate $\tau_{w a l l}$ and $y^{+}$using Equations (19) and (11).

c. Fix the values of $K$ and $\epsilon$ at the first grid points using Equations (15) and (16). Solve the turbulence equations.

d. Calculate $q_{w a l l}$ using Equation (23).

e. Calculate $U_{c}$ and $U_{c}^{+}$using Equations (12) and (10).

f. Update $\mu_{t}$ using Equation (18). g. Update $\alpha$ using Equation (22).

h. Update $g_{22}, g_{23}$ and $g_{24}$ using Equation (27) or (29); or update $g_{12}, g_{13}$ and $g_{14}$ using Equation (33) or (34). Solve the Navier-Stokes equations.

i. Return to step b with updated field values.

The sequence of steps $b$ to $i$ are repeated until the calculation converges.

Note that by definition, the turbulent eddyviscosity $\mu_{t}$ is zero at the wall, such as in the case of the low Reynolds number turbulence models. When using the wall functions, Equation (18) introduces the effective turbulent viscosity which is defined at the wall in the turbulence module. Therefore in post-processing, the wall friction coefficient $C_{f}$ can be calculated in the same way as for laminar flows, except that the molecular viscosity $\mu$ is replaced by the turbulent viscosity $\mu_{t}$ at the wall.

\section{Module Usage}

The present turbulence module (version 2.0) is written based on the NPARC version 2.2. The following are those parts of the code which may require user's attention when using it.

\subsection{Module}

To facilitate identification, all the subroutine names in the module start with $\mathrm{CM}$. In order to use the module, the user only needs to call its three subroutines: CMA0, CMALL and CMVRHS, in the NPARC code.

Subroutine CMAO. This subroutine transfers from NPARC to the module the parameters to define flow, geometric and boundary conditions. In addition, it has the following user-specified parameters:

FDEFER - Blending factor in the convection scheme. Its value may vary from 0 to 1 with the limiting value 0 for the first-order accurate upwind and 1 for the second-order accurate HLPA scheme. The solution tends to be more stable, but also more diffusive when this factor is reduced.

JTDMA, KTDMA - The value of these integer parameters determines whether to use the TDMA solution algorithm in J- or $\mathrm{K}$-direction ( 1 does; 0 doesn't). Currently, they are set to

$$
\begin{aligned}
& \text { JTDMA }=1 \\
& \text { KTDMA }=1
\end{aligned}
$$

BDMAX(i), BDMIN(i) - Upper and lower bounds for the values of $K(i=1), \epsilon(i=2)$ and $\mu_{t}(i=3)$. 
These bounds are introduced for numerical purposes only, that is, to prevent the corresponding turbulence quantities from becoming negative or abnormally large during the solution process. Currently, they are set to

$$
\begin{aligned}
& \operatorname{BDMAX}(1)=1.0 \mathrm{E}+6 \\
& \operatorname{BDMAX}(2)=1.0 \mathrm{E}+6 \\
& \operatorname{BDMAX}(3)=1.0 \mathrm{E}+4 \\
& \operatorname{BDMIN}(1)=1.0 \mathrm{E}-8 \\
& \operatorname{BDMIN}(2)=1.0 \mathrm{E}-8 \\
& \operatorname{BDMIN}(3)=1.0 \mathrm{E}-3
\end{aligned}
$$

which should cover a wide range of the physically meaningful values of $K, \epsilon$ and $\mu_{t}$. It is to be noted that these values are only valid for the nondimensional turbulence quantities, as defined in the NPARC code.

RELAX(i) - Under-relaxation factors for $K(i=1)$ and $\epsilon(i=2)$. Currently, they are set to

$$
\operatorname{RELAX}(1)=0.8
$$$$
\operatorname{RELAX}(2)=0.8
$$

Should instability occur, try to reduce these values.

Subroutine CMALL. This is the main subroutine to control the solution sequence in the module. The array variable VIST is the turbulent viscosity $\mu_{t}$ which is needed in NPARC for calculating turbulent flows. The array variables TE, ED and YPS are $K, \epsilon$ and $y^{+}$, respectively, which can be used for post-processing. Normally, there is no need for user to change this subroutine.

Subroutine CMVRHS. This subroutine which is the counterpart of the subroutine VISRHS in NPARC is for introducing the wall function modifications into the right-hand side viscous flux terms. There is no need for user to change this subroutine.

\subsection{NPARC}

The authors have made all the modifications necessary for NPARC to use the module. The following shows where these modifications are in NPARC. All the alterations are marked between $\mathrm{C}<<$ and $\mathrm{C}>>$ in the code.

Namelist TURBIN. The integer parameter IMUTR2 is used to select the turbulence models in the module with

$$
\begin{aligned}
\text { IMUTR2 }=101 & \text { Chien model } \\
102 & \text { Shih-Lumley model } \\
103 & \text { CMOTT model }
\end{aligned}
$$

A new integer parameter MWALF is introduced to select the near-wall approach with
MWALF $=0$ low Reynolds number approach

1 wall function approach

Correspondingly, a new statement is added

in the include file NPARC.INC:

COMMON/CMOTT/MWALF

and in the subroutine TURBIN:

CALL NLGETI('MWALF',MWALF).

Subroutines FIIT1, FILT2, FILTER. An array FAV01 has been introduced into each of these subroutines to eliminate the artificial viscosity in the near-wall region.

Subroutines INITIA, WREST. The model identifier ITURB for each $K-\epsilon$ turbulence model in the module is given an integer value greater than 100. To reflect this expanded choice for turbulence models, the read and write statements for the turbulent quantities in these two subroutines are modified as

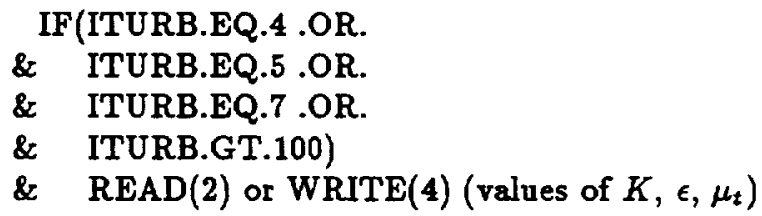

Subroutine MUTURB. The subroutines CMA0 and CMALL of the module are called here.

Subroutine STPF2D. The subroutine CMVRHS of the module is called here.

\section{Application}

Turbulent boundary layer flow over a flat plate with zero pressure gradient was selected as the first test case for code validation. The solution of the wall function approach (WF) was compared with both the experimental data (Exp) of Wieghardt and Tillmann (1951) and the solution of the low Reynolds number approach (LR). The Shih-Lumley model was used in this test. Note that the high Reynolds number version of this model is of the same form as the standard $K-\epsilon$ model of Launder and Spalding (1974). Figure 2 shows the flow geometry and boundary conditions used in the calculation. For the wall function approach, grid points were $111 \times 55$, the first grid points above the wall had the $y^{+}$value of 60 and 14 grid points in the $x$-direction were located before the leading edge of the flat plate. For the low Reynolds number approach, grid points were $111 \times 81$ with the distribution of $x$-points being the same as in the wall function case and the first $y^{+}$ 
being 0.3 . Since the NPARC code is for compressible flows while the experiment (Wieghardt and Tillmann, 1951) to be compared was for incompressible flows, a freestream Mach number of 0.2 was chosen. The reference Reynolds number $R_{e}$ was $6.9 \times 10^{6}$. All calculations started from an initial field with $U=0.2, V=0, \mu_{t}=1, K=0.005$ and $\epsilon=0.09 R_{e} \rho K^{2} / \mu_{t}$. The influence of the artificial viscosity originally implemented in the NPARC code was first examined. This was done by simply reducing the coefficient DIS4 of the 4th-order artificial viscosity. For this particular flow, the 2nd-order artificial viscosity was inactive due to the zero pressure gradient condition. The results are shown in Figure 3 for a streamwise mean velocity profile at a given location and in Figure 4 for the wall friction coefficient. It can be seen that the artificial viscosity with DIS4 $=0.6$ totally spoiled the solution of the wall function approach and very good results were obtained only when DIS4 was reduced to 0.001 . Although reducing the artificial viscosity can significantly improve the prediction in this test case, this method is generally unfeasible, because the artificial viscosity will also overly be reduced in places where certain amount is needed. In the near-wall region, the turbulent viscosity prevails, which is enough to stabilize the calculation. Satisfactory results were obtained, as shown in Figures 5 and 6 , when turning off the artificial viscosity only in the near-wall area while having DIS $4=0.6$ elsewhere. Regarding the computational cost, 1000 iterations on the Cray YMP computer took 177 seconds for the wall function approach and $\mathbf{2 6 2}$ seconds for the low Reynolds number approach.

\section{Conclusions}

The compressible wall functions based on the Van Driest transformation have been implemented in the turbulence module developed for the NPARC code. The details of the numerical implementation are reported.

The module is validated against the incompressible flow over a flat plate. Very good results have been obtained. It is found that the unrealistic artificial viscosity near the wall has a big influence on the solution of the wall function approach. The simplest way to eliminate this influence, as used in this work, is to turn off the artificial viscosity in the near-wall region. The module has to undergo further tests be fore fully establishing its computational capability.

This work is only restricted to the simple form of the wall functions. Refined forms have been available which account for pressure gradient effects and mass flow through the wall or the effects of the viscous sublayer. Their implementation will be a subject of further development of the module.

\section{References}

1. Chien, K.Y., "Predictions of channel and boundary-layer flows with a low Reynolds number turbulence model," AIAA Journal, Vol.20, 1982, pp.33-38.

2. Chitsomboon, T., "Effects of artificial viscosity on the accuracy of high Reynolds number $K-\epsilon$ turbulence model," NASA TM 106781, 1994.

3. Cooper, G.K., and Sirbaugh, J.R., "PARC code: theory and usage," AEDC-TR-89-15, Arnold Engineering Development Center, 1989.

4. Cooper, G.K., and Sirbaugh, J.R., "The PARC distinction: a practical flow simulator," AIAA 90-2002, 1990.

5. Huang, P.G., and Coakley, T.J., "Calculations of supersonic and hypersonic flows using compressible wall functions, ${ }^{n}$ presented at the 2nd Int. Symp. on Turbulence Modeling and Measurement, 1993, Florence, Italy.

6. Launder, B.E., and Spalding, D.B., "The numerical computation of turbulent flows," Computer Methods in Applied Mechanics and Engineering, Vol.3, 1974, pp.269-289.

7. Shih, T.-H., and Lumley, J.L., "Kolmogorov behavior of near-wall turbulence and its application in turbulence modeling," Comp. Fluid Dyn., Vol.1, 1993, pp.43-56.

8. Van Driest, E.R., "Turbulent boundary layer in compression fluids," J. Aeronaut. Sci., Vol.18, 1951, pp.145-160.

9. Viegas, J.R., Rubesin, M.W., and Horstman, C.C., "On the use of wall functions as boundary conditions for two dimensional separated compressible flows," AIAA 85-0180, presented at the AIAA 23rd Aerospace Sciences Meeting, January 1985 , Reno, Nevada.

10. Wieghardt, K., and Tillmann, W., "On the turbulent friction layer for rising pressure," NACA TM 1314, 1951.

11. Yang, Z., Georgiadis, N.J., Zhu, J. and Shih, T.-H., "Calculations of inlet/nozzle flows using a new $K-\epsilon$ model," AIAA 95-2761, presented at the 31st AIAA/ASME/SAE/ASEE Joint Propulsion Conference and Exhibit, July 1995, San Diego, CA.

12. Zhu, J., and Shih, T.-H., "A turbulence module for the NPARC code," AIAA 95-2612, presented at the 31st AIAA/ASME/SAE/ASEE Joint Propulsion Conference and Exhibit, July 1995, San Diego, CA. 


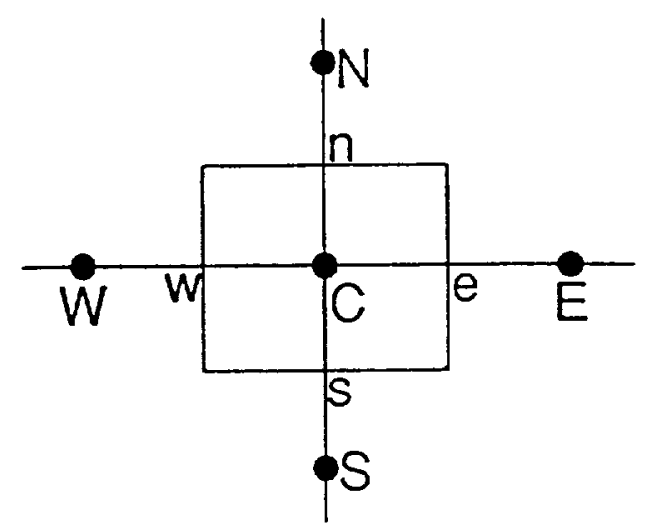

Fig.1 A typical control volume centered at node C and its neighbouring nodes

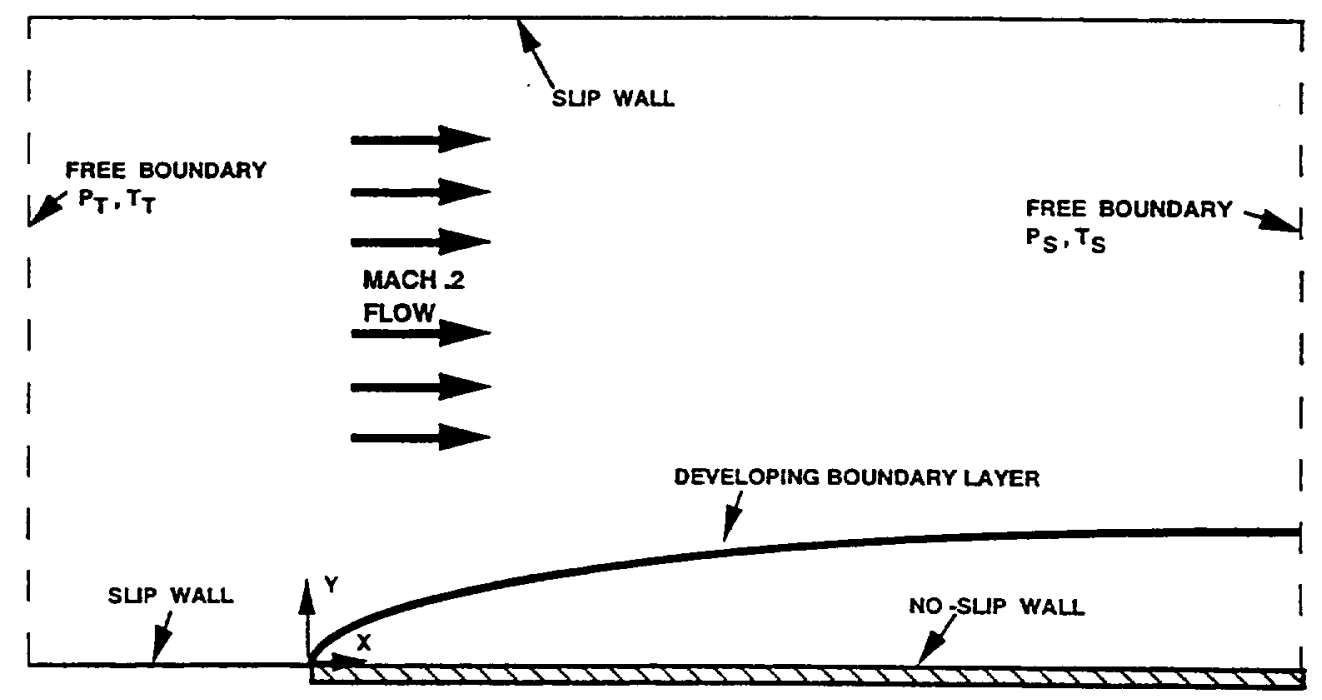

Fig.2 Geometry of solution field 


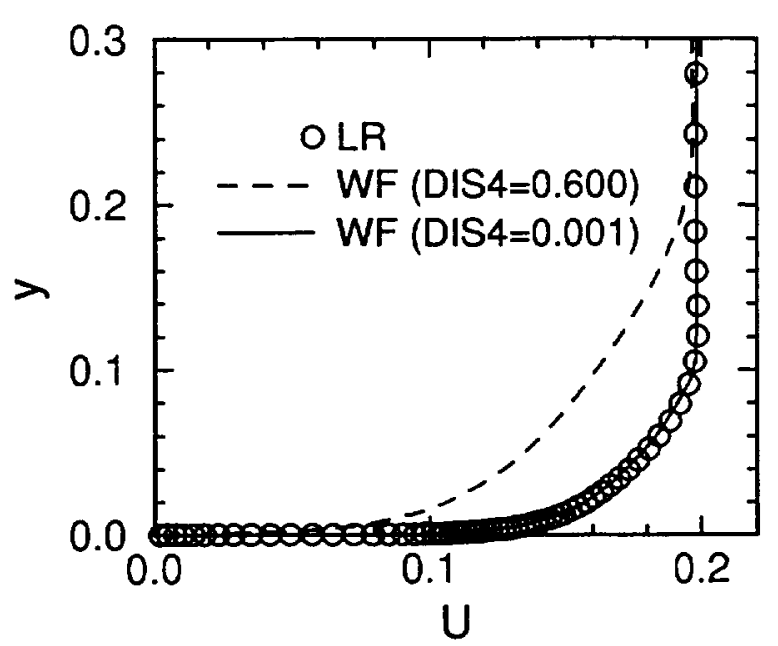

Fig.3 Influence of artificial viscosity on U-velocity profile

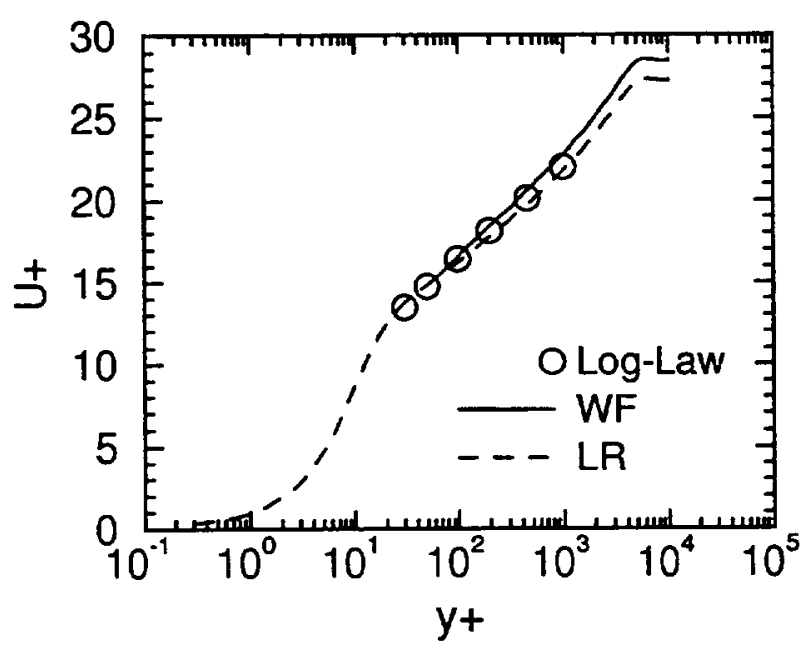

Fig.5 U-velocity profile

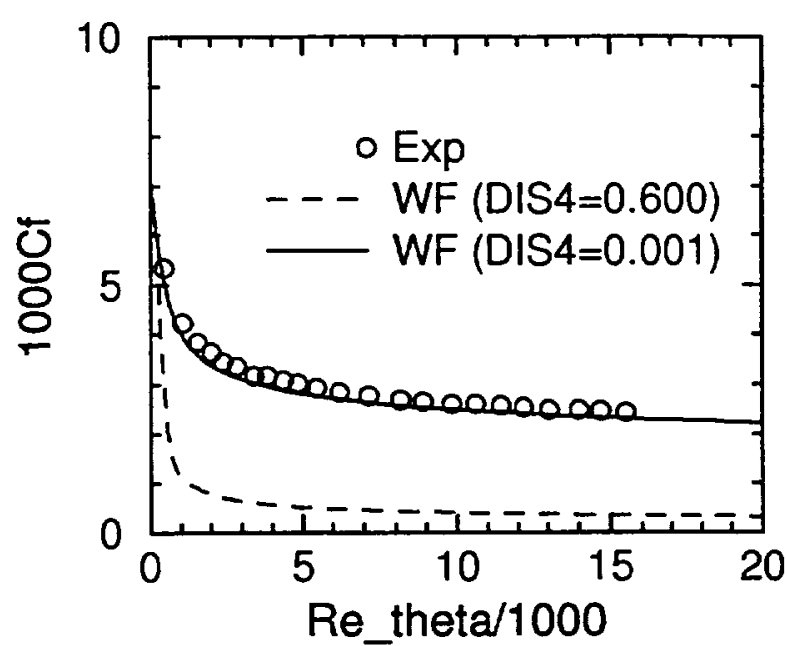

Fig.4 Influence of artificial viscosity on wall friction coefficient

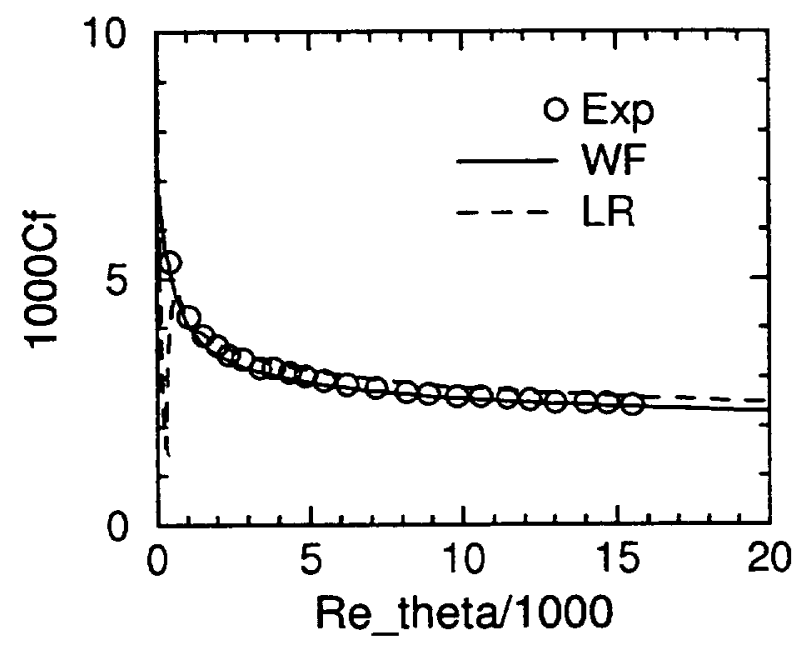

Fig. 6 Wall friction coefficient 
Public reporting burden for this collection of information is estimated to average 1 hour per response, including the time for reviewing instructions, searching existing data sources, gathering and maintaining the data needed, and completing and reviewing the collection of information. Send comments regarding this burden estimate or any other aspect of this collection of information, including suggestions tor reducing this burden, to Washington Headquarters Services, Directorate lor Information Operations and Reports, 1215 Jefferson Davis Highway, Suite 1204, Arlington, VA 22202-4302, and to the Office of Management and Budget, Papenwork Reduction Project (0704-0188), Washington, DC 20503.
1. AGENCY USE ONLY (Leave blank)
2. REPORT DATE
3. REPORT TYPE AND DATES COVERED

4. TITLE AND SUBTTTLE

August 1997

Contractor Report

An NPARC Turbulence Module With Wall Functions

5. FUNDING NUMBERS

WU-523-36-13-00

6. AUTHOR(S)

NCC $3-370$

J. Zhu and T.-H. Shih

7. PERFORMING ORGANIZATION NAME(S) AND ADDRESS(ES)

Institute for Computational Mechanics in Propulsion

22800 Cedar Point Road

Cleveland, Ohio 44142

8. PERForming ORGANIZATION

REPORT NUMBER

9. SPONSORINGMONITORING AGENCY NAME(S) AND ADDRESS(ES)

10. SPONSORINGMONITORING AGENCY REPORT NUMBER

National Aeronautics and Space Administration

Lewis Research Center

Cleveland, Ohio 44135-3191

NASA CR-204142

ICOMP-97-09; CMOTT-97-04

AIAA-96-0382

11. SUPPLEMENTARY NOTES

Prepared for the 34th Aerospace Sciences Meeting and Exhibit, Reno, Nevada, January 15-18, 1996. ICOMP Program

Director, Louis A. Povinelli, organization code 5800, (216) 433-5818.

12a. DISTAIBUTION/AVAILABILITY STATEMENT

12b. DISTRIBUTION CODE

Unclassified - Unlimited

Subject Category 34

This publication is available from the NASA Center for AeroSpace Information, (301) 621-0390.

13. ABSTRACT (Meximum 200 words)

The turbulence module recently developed for the NPARC code has been extended to include wall functions. The Van Driest transformation is used so that the wall functions can be applied to both incompressible and compressible flows. The module is equipped with three two-equation $K$ - $\varepsilon$ turbulence models: Chien, Shih-Lumley and CMOTT models. Details of the wall functions as well as their numerical implementation are reported. It is shown that the inappropriate artificial viscosity in the near-wall region has a big influence on the solution of the wall function approach. A simple way to eliminate this influence is proposed, which gives satisfactory results during the code validation. The module can be easily linked to the NPARC code for practical applications.

14. SUBJECT TERMS

Turbulence modeling; Wall functions; Compressible flow; Incompressible flow

17. SECURITY CLASSIFICATION OF REPORT

Unclassified
18. SECURITY CLASSIFICATION OF THIS PAGE

Unclassified
19. SECURTY CLASSIFICATION OF ABSTRACT Unclassified 16. PRICE CODE A03

15. NUMBER OF PAGES

$\mathrm{A} 03$

20. LIMITATION OF ABSTRACT 